\title{
Assessment of Full-Eye Response to Osmotic Stress in Mouse Model In Vivo Using Optical Coherence Tomography
}

\author{
Yang Ni, ${ }^{1}$ Baisheng Xu, ${ }^{2}$ Lan Wu, ${ }^{1}$ Chixin Du, ${ }^{2}$ Bo Jiang, ${ }^{2}$ Zhihua Ding, ${ }^{1}$ and Peng Li ${ }^{1}$ \\ ${ }^{1}$ State Key Lab of Modern Optical Instrumentation, Department of Optical Engineering, Zhejiang University, Hangzhou, \\ Zhejiang 310027, China \\ ${ }^{2}$ Department of Ophthalmology, First Affiliated Hospital, College of Medicine, Zhejiang University, Hangzhou, Zhejiang 310027, China
}

Correspondence should be addressed to Chixin Du; duchixin@126.com and Peng Li; peng_li@zju.edu.cn

Received 26 June 2015; Revised 11 September 2015; Accepted 13 September 2015

Academic Editor: Ireneusz Grulkowski

Copyright (c) 2015 Yang Ni et al. This is an open access article distributed under the Creative Commons Attribution License, which permits unrestricted use, distribution, and reproduction in any medium, provided the original work is properly cited.

\begin{abstract}
$\mathrm{NaCl}$ based solutions were applied as osmotic stress agents to alter the hydration state of the mouse eye. Full-eye responses to these osmotic challenges were monitored in vivo using a custom-built optical coherence tomography (OCT) with an extended imaging range of $12.38 \mathrm{~mm}$. Dynamic changes in the mouse eye were quantified based on the OCT images using several parameters, including the central corneal thickness (CCT), the anterior chamber depth (ACD), the crystalline lens thickness (LT), the cornearetina distance (CRD), the iris curvature (IC), and the lens scattering intensity (LSI). Apparent but reversible changes in the morphology of almost all the ocular components and the light transparency of the lens are exhibited. Particularly, the ocular dehydration induced by the hypertonic challenges resulted in a closing of the iridocorneal angle and an opacification of the lens. Our results indicated that the ocular hydration is an important physiological process which might be correlated with various ocular disorders, such as dry eye, cataract, and angle-closure glaucoma, and would affect the biometry and imaging of the eye. OCT uniquely enables the comprehensive study of the dynamic full-eye responses to the ocular hydration in vivo.
\end{abstract}

\section{Introduction}

The ocular hydration is an important physiological process. The process might have a significant correlation with various ocular disorders, such as dry eye [1-3], cataract [4-8], and glaucoma [9-11]. It may also affect the accuracy of the measuring tools in ophthalmology $[12,13]$. Intensive efforts have been dedicated to the characterization and evaluation of the fluid transport mechanism [14-17] in ocular. The early studies were mainly based on the in vitro experiments and focused on a single component (cornea, lens, etc.) [1820]. Ruberti and Klyce examined a trans-endothelial fluid transport system in response to $\mathrm{NaCl}$ osmotic challenges in the excised rabbit cornea using an automatic scanning specular microscope for corneal thickness measurement [18]. Marcantonio et al. investigated the effect of osmotic stress on lens opacification and crystallin loss using the organ culture of bovine lenses [19]. Optical coherence tomography (OCT) is a powerful noninvasive, three-dimensional (3D), real-time imaging modality with high axial resolution. As the advance of long imaging range, OCT is capable of full-eye imaging in mouse [21-24] and even in human with VSCEL swept source [25]. OCT has been used for noninvasive monitoring and quantification of diffusion of different analytes in sclera and cornea in vivo $[26,27]$. Hosseini et al. reported an in vivo study of the corneal response to the dehydration stress after topical administration of hypertonic agents and prolonged surface evaporation of the cornea [28]. The osmotic stress would lead to a fluid transport between the cornea and the aqueous humor. Consequently, dynamic changes in intraocular pressure (IOP), crystalline lens, and retina should also be exhibited. Therefore, quantitatively assessing the fulleye response to osmotic stress in vivo would be of great importance to comprehensively understanding the influence of the ocular hydration.

In this pilot study, osmotic stress was induced by topical administration of the drops of $\mathrm{NaCl}$ based stress agents in mouse model in vivo. The dynamic changes of the cornea, the crystalline lens, the retina, and the iris were monitored simultaneously using a custom-built OCT system with an 
TABLE 1: Study procedures of Groups I-IV.

\begin{tabular}{|c|c|c|c|}
\hline Group & Phase 1 & Phase 2 & Phase 3 \\
\hline \multirow{2}{*}{ Control } & \multicolumn{3}{|c|}{ Isotonic $\left(250^{*}\right)$} \\
\hline & \multicolumn{3}{|c|}{60 mins, $1 / 5$ times per min } \\
\hline \multirow{2}{*}{ I } & & Hypertonic $\left(1000^{*}\right)$ & \\
\hline & & 13 mins, 1 time per min & Isotonic $\left(250^{*}\right)$ \\
\hline \multirow{2}{*}{ II } & & Hypertonic $\left(500^{*}\right)$ & 40 mins, $1 / 5$ times per min \\
\hline & Isotonic $\left(250^{*}\right)$ & 13 mins, 1 time per min & \\
\hline \multirow{2}{*}{ III } & 10 mins, $1 / 5$ times per $\min$ & Hypotonic $\left(100^{*}\right)$ & Isotonic $\left(250^{*}\right)$ \\
\hline & & 24 mins, $1 / 3$ times per min & 24 mins, $1 / 3$ times per min \\
\hline \multirow{2}{*}{ IV } & & Hypertonic $\left(1000^{*}\right)$ & Hypotonic $\left(100^{*}\right)$ \\
\hline & & 20 mins, single time & 20 mins, single time \\
\hline
\end{tabular}

${ }^{*}$ Osmolality with unit of $\mathrm{mOsmol} / \mathrm{kg}$.

extended imaging range and high resolution. The feasibility of characterizing the full-eye response to osmotic challenge was demonstrated in vivo in mouse model by OCT.

\section{Materials and Methods}

2.1. System Setup. A custom-designed SD-OCT system was built for this study, which has been detailed in [29]. Briefly, a superluminescent diode (SLD) with a central wavelength of $850 \mathrm{~nm}$ and a spectral bandwidth of $100 \mathrm{~nm}$ was used as a broadband light source, offering a measured axial resolution of $\sim 4.3 \mu \mathrm{m}$ in air. In the sample arm, an objective lens (focal length of $75 \mathrm{~mm}$ ) was used to focus the probing light beam on the sample, yielding a measured lateral resolution of $\sim 40 \mu \mathrm{m}$. The spectral interference fringe between the light from the reference and sample arms was recorded by a high-speed spectrometer, equipped with a high-speed line scan CMOS camera (Basler, Ahrensburg, Germany, Sprint spL4096-140k) providing $70 \mathrm{kHz}$ line scan rate. The spectrometer had a spectral resolution of $0.029 \mathrm{~nm}$ providing a measured imaging range of $\sim 6.19 \mathrm{~mm}$ in air at half Fourier space. To fully utilize the output Fourier space for imaging, we employed a full-range complex method by utilizing the X-scanner to modulate spatial interferograms and thus removed the complex conjugate ambiguity artifact [21, 3033 , delivering a $\sim 12.38 \mathrm{~mm}$ maximal imaging range and a $6 \mathrm{~dB}$ sensitivity rolling-off within the central $\sim 6 \mathrm{~mm}$ range in air. To facilitate the implementation of full-range complex method and achieve high definition in the cross-sectional images, the scanning protocol was configured with 1500 lines per frame and a B-scan rate of 46 frames per second for $3 \mathrm{D}$ raster scanning. During the OCT scanning, the mouse eye was positioned at the central $\sim 6 \mathrm{~mm}$ range for the best imaging quality of full eye. The system dynamic range was measured at $\sim 50 \mathrm{~dB}$ at the depth-position of $0.5 \mathrm{~mm}$ with an incident optical power of $2.5 \mathrm{~mW}$ in the sample.

2.2. Animal Protocol. Twenty-five C57BL/6 wild type mice at 16 to 18 weeks of age were obtained from the laboratory animal center at Zhejiang University (Hangzhou, China). All animals were raised and treated in compliance with the Association for Research in Vision and Ophthalmology (ARVO) Statement for the Use of Animals in Ophthalmic and
Vision Research. The study was approved by the Institutional Animal Care and Ethics Committee of Zhejiang University.

Animals were all anesthetized 10 minutes before each experiment by intraperitoneal injection of $4 \%$ chloral hydrate $(10 \mathrm{~mL} / \mathrm{kg})$. In the meantime, pupils were dilated with $0.5 \%$ tropicamide $/ 0.5 \%$ phenylephrine mixed eye-agent (Mydrin$\mathrm{P}$, Santen, Osaka, Japan). The mice were placed in a cylindrical holder and mounted on a multiaxis stage in front of the scanning probe during the experiments. A crosshair scanning pattern was designed for the fine alignment between the eye axis and the scanning beam by simultaneously examining the OCT cross-sections in the horizontal and vertical meridians.

2.3. Study Procedure. Saline $(\mathrm{NaCl})$ solutions with different concentrations served as stress agents in this study. The osmolality of the stress agents was 1000, 500, 250, and $100 \mathrm{mOsmol} / \mathrm{kg}$ (denoted as Drop-1000, Drop-500, Drop250, and Drop-100, resp.) which were verified by an osmometer (OSA-22; Nikkiso, Tokyo, Japan). Among them, Drop250 induces minimal changes of the ocular tissue (referring to Figure 2(a)), and it was used as the isotonic agents in this study. To induce osmotic challenges, drops of the stress agents were applied to the mouse cornea in vivo at the rate of 1 drop per 5 mins. The 25 mice were equally divided into five groups (Control and I-IV), and one eye of each mouse was randomly selected for OCT imaging, as described in Table 1. Groups I and II were designed for hypertonic challenges with different levels (250-1000/500-250 mOsmol/kg); that is, the cornea was sequentially treated with isotonic Drop250 for 10 mins, hypertonic Drop-1000/500 for 13 mins, and isotonic Drop-250 again for 40 mins. In the meantime, OCT imaging was performed with highest frequency of 1 time/min at the hypertonic phase to follow the acute response. Group III was conducted for hypotonic challenges (250-100$250 \mathrm{mOsmol} / \mathrm{kg}$ ); that is, the cornea was sequentially treated with drops of isotonic Drop-250 for 10 mins, hypotonic Drop100 for 24 mins, and isotonic Drop-250 again for 24 mins. Group IV was optimized for monitoring the response of the iris morphology to the osmotic challenges (250-1000$100 \mathrm{mOsmol} / \mathrm{kg}$ ); that is, the cornea was sequentially treated with drops of isotonic Drop-250 for 10 mins, hypertonic Drop-1000 for 20 mins, and hypotonic Drop-100 for 20 mins. Besides, all the baseline OCT measurements were performed 
TABLE 2: Definition of parameters.

\begin{tabular}{ll}
\hline Parameters & Definition \\
\hline CCT & Distance from corneal surface to corneal endothelium \\
LCD & Distance from corneal endothelium to anterior crystalline lens surface \\
CRD & Distance from anterior crystalline lens surface to the posterior crystalline lens surface \\
LSI & Distance from corneal endothelium to the inner limiting membrane of the retina \\
IC & $\begin{array}{l}\text { Average backscatter intensity of the lens from the anterior capsular area to the anterior nuclear area along the AP axis } \\
\text { Maximum perpendicular distance between the iris pigment epithelium and the line connecting the iris root and the } \\
\text { most peripheral point of contact between the iris and lens. }\end{array}$ \\
\hline
\end{tabular}

5 times prior to each osmotic challenges. The time range of the $\mathrm{NaCl}$ agent administration for Phase 2 and Phase 3 was mainly determined by the response time that the ocular tissue required to reach a steady state. In this study, the steady state is defined as the change rate of the ocular tissue has decreased to less than $1 \%$ of the initial rate.

2.4. Data Processing. To quantify the dynamic changes, a custom semiautomatic algorithm was developed for biometric analysis. The cross-sectional slice including the corneal vertex location was extracted from each $3 \mathrm{D}$ dataset at the horizontal meridian. Optical distortion in the cross-section was corrected based on Snell's principle [34], in which the refractive indices $n$ of $1.40,1.33,1.57$, and 1.33 were used for cornea, aqueous humor, crystalline lens, and vitreous cavity, respectively [35]. According to our OCT measurements, the refractive indices $n$ of Drop-1000, Drop-500, Drop-250, and Drop-100 are $1.344,1.342,1.337$, and 1.333 , respectively, and the maximal difference between them is less than $0.82 \%$. Thus, the refractive indices of ocular tissue should not change significantly in response to these osmotic agents and were assumed to be constant and independent of the hydration level in our study. Four parameters were measured along the anteroposterior (AP) axis [21, 35], including the central corneal thickness (CCT), the anterior chamber depth (ACD), the crystalline lens thickness (LT), and the cornea-retina distance (CRD). Moreover, the lens scattering intensity (LSI) and the iris curvature (IC) were measured to evaluate the optical property of the lens and the morphologic change of the iris, respectively. The definitions of the parameters were summarized in Table $2[21,35]$. 3D OCT imaging of a commercially available model eye (OEMI-7, Ocular Instruments Inc., Bellevue, WA) was performed 5 times to validate the accuracy and the repeatability of the developed algorithm. The anterior segment parameters of the model eye were measured and matched well with the values provided by the manufacturer, as shown in Table 3.

\section{Results and Discussions}

Figure 1(a) is a 3D rendering of the OCT full-eye imaging in the mouse model, and Figure 1(b) is a representative OCT cross-section in the horizontal meridian. As illustrated in Figure 1(b), the primary ocular components can be clearly visualized in the wide field OCT imaging, including cornea,
TABLE 3: OCT measurement of model eye.

\begin{tabular}{lcc}
\hline Parameters & $\begin{array}{c}\text { OCT values } \pm \text { STD } \\
(\mathrm{mm})\end{array}$ & $\begin{array}{c}\text { Manufacturer values } \\
\pm \text { tolerance }(\mathrm{mm})\end{array}$ \\
\hline CCT & $0.46 \pm 0.01$ & $0.55 \pm 0.13$ \\
ACD & $3.04 \pm 0.01$ & $3.03 \pm 0.13$ \\
LT & $3.85 \pm 0.01$ & $3.9 \pm 0.51$ \\
\hline
\end{tabular}

iris, crystalline lens, and retina, based on which the biometric measurements could be readily performed using the processing algorithms. As marked by the yellow rectangular region in Figure 1(b), a serial of clips centered at the AP axis are extracted from different phases of Group I and display along the time dimension in Figure 1(c). All these clips are aligned along the posterior surface of the cornea in the depth direction. Apparently, due to the dehydration induced by the hypertonic challenge, a thinning of the cornea, decrease of ACD and CRD, and an obvious increase of the lightscattering of the anterior lens are exhibited. The reversibility of all these changes can be visualized after the return to isotonicity. In addition, it is shown that the scattering property of the anterior lens would change gradually from the surface towards the center in both the dehydration and the rehydration phases. In the transparent lens, the collagen fibers are arranged in order $[36,37]$. The osmotic stress induces the water movement in the lens, and the normal distribution of fibers is changed. The disordered arrangement of fibers results in the increase of light scattering in lens and the loss of transparency $[36,37]$. The gradual change of the lens scattering reveals the spatial characterization of the hydration state and ultrastructure in lens.

The dynamic responses of the cornea, the lens, and the retina to the osmotic challenges were further quantified and plotted in Figure 2. Of all the mice used in the control group and Groups I-III, the baselines (mean \pm standard deviation) of the CCT, ACD, LT, and CRD are $119 \pm 7 \mu \mathrm{m}, 404 \pm 9 \mu \mathrm{m}$, $1860 \pm 14 \mu \mathrm{m}$, and $3080 \pm 18 \mu \mathrm{m}$, respectively. To eliminate individual differences, the relative changes of all the parameters were normalized by the corresponding baseline values for each mouse. Generally speaking, the cornea, the lens, and the retina exhibited no appreciable change in the first isotonic phases, then responded with different magnitudes in the subsequent challenge phases, and gradually returned to the baselines in the reversal phases, while no meaningful change was observed in the control group (the mean standard deviation of the fluctuations $< \pm 1.5 \%$ ). 


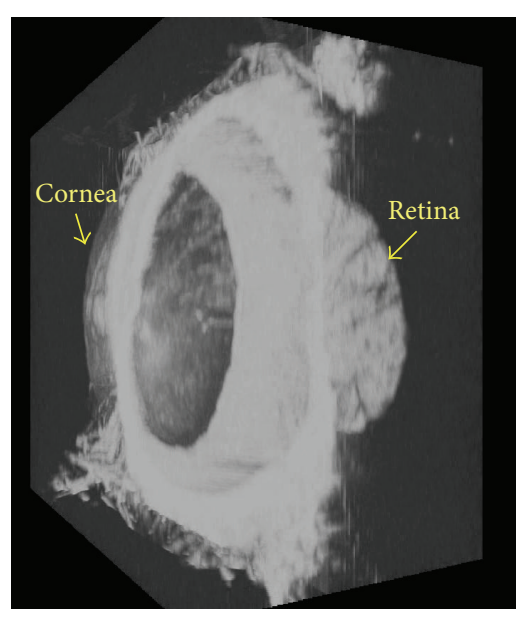

(a)

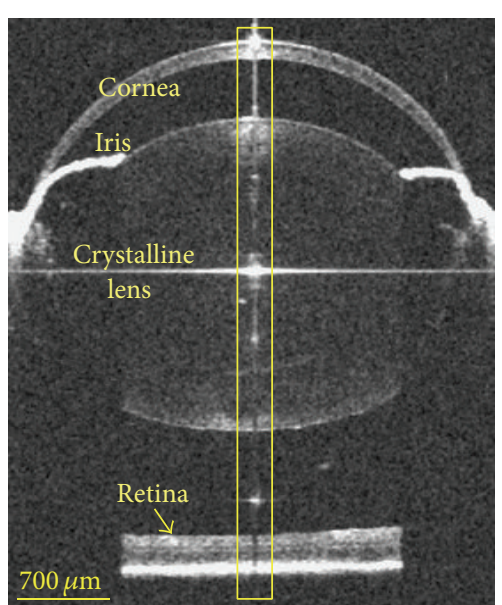

(b)

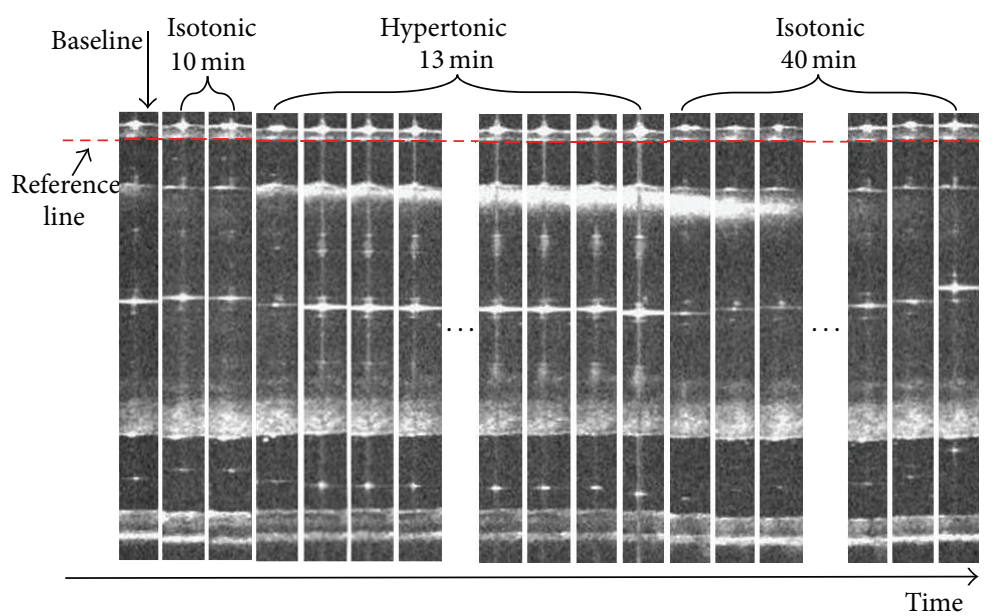

(c)

FIGURE 1: OCT full-eye imaging and dynamic response to hypertonic challenge and reversal in mouse model. (a) Three-dimensional rendering of the OCT full-eye imaging of mouse. (b) Representative OCT cross-section image in the horizontal meridian. As marked by the yellow rectangular region in (b), a serial of clips around the corneal vertex reflection line were extracted and displayed along the time dimension to demonstrate the dynamic response in (c). In the depth direction, all clips were aligned along the posterior cornea.

The hypertonic challenge could lead to a global dehydration of the eyeball. Under the hypertonic Drop-1000 challenge (Group I), as reported in Figure 2(b), the corneal thickness (CCT) exhibited a rapid and exponential thinning of up to $20.7 \%$. The anterior chamber depth (ACD) showed a maximal decrease of $11.5 \%$. The relative distance between the cornea and the retina, that is, the cornea-retina distance (CRD), presented a maximal shortening of $2.3 \%$. The crystalline lens presented no considerable change in its thickness (LT). However, the lens dehydration induced a $28.8 \%$ increase of the optical scattering (LSI), resulting in an optical opacity of the lens. The mouse eyes treated with hypertonic Drop-500 challenge (Group II) exhibited a similar changing tendency as those with Drop-1000 but with a decreased rate and magnitude, as shown in Figure 2(c). Compared with the hypertonic challenges, the hypotonic Drop-100 challenge (Group III) induced an opposite tendency at a slower changing rate of the mouse eye, as depicted in Figure 2(d). No meaningful change of the lens scattering property was observed in Group III.
Figure 3 shows the representative iris responses to the osmotic challenges. In the control group, the iris curvatures (IC) were 29.1, 30.2, and 31.8 at times 0,30 , and $60 \mathrm{~min}$, respectively, and no apparent change of the iris curvature was measured. The ICs were $28.6 \mu \mathrm{m}, 48.2 \mu \mathrm{m}$, and $-24.6 \mu \mathrm{m}$ in the isotonic, hypertonic, and hypotonic phases, respectively. As we know, there is a close correlation between iris configuration and IOP [38]. Accordingly, most likely due to the dehydration of the cornea and anterior chamber during the hypertonic challenge, there would be a decrease of the IOP in the anterior chamber, generating a transient outward force in the posterior chamber and an outward bowing of the iris (convexity). In contrast, the hypotonic challenge would lead to a transient inward force in the anterior chamber, corresponding to an inward bowing of the iris (concavity).

In this study, it is observed from the OCT imaging that the osmotic challenges lead to reversible changes of the ocular morphology and the light scattering property of the lens. In particular, the opacification of the lens (Figure 1) and the 


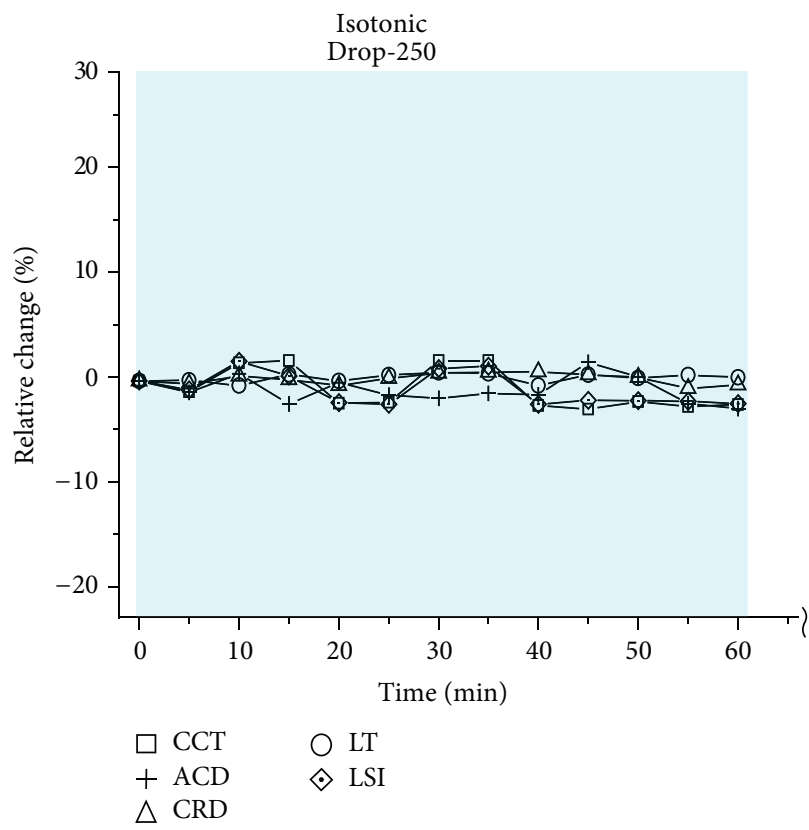

(a)

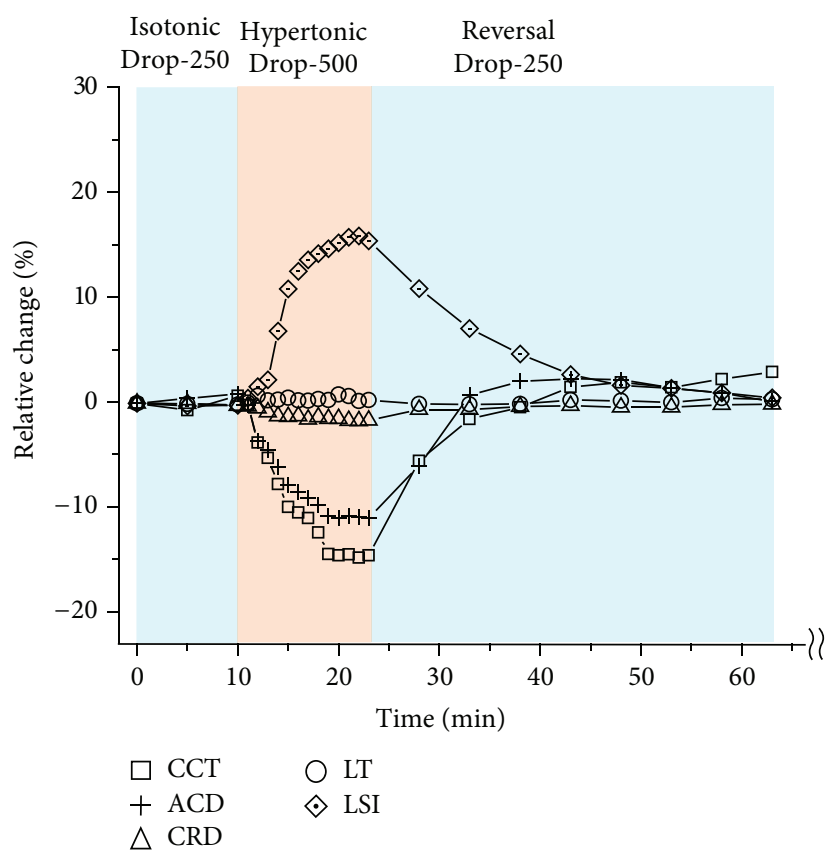

(c)

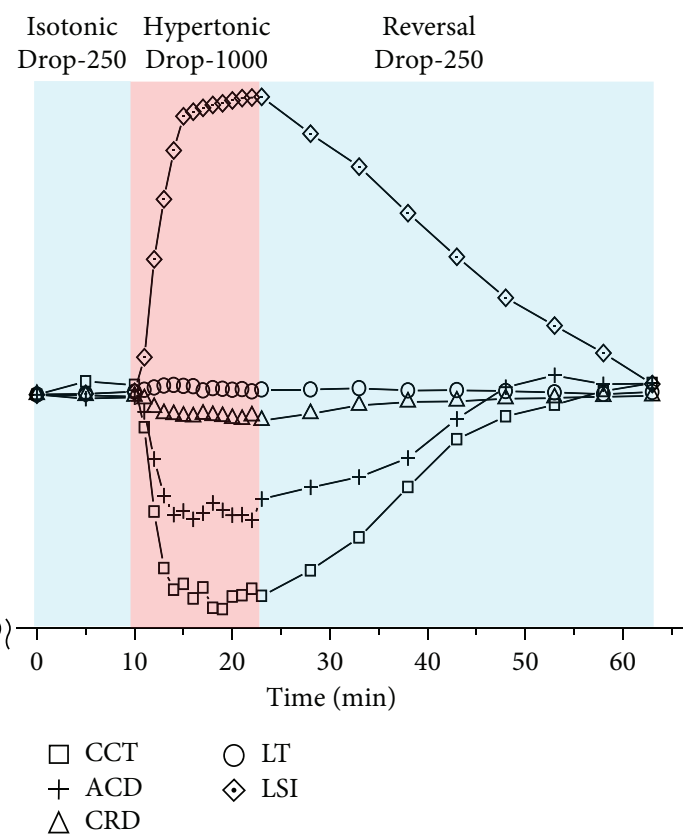

(b)

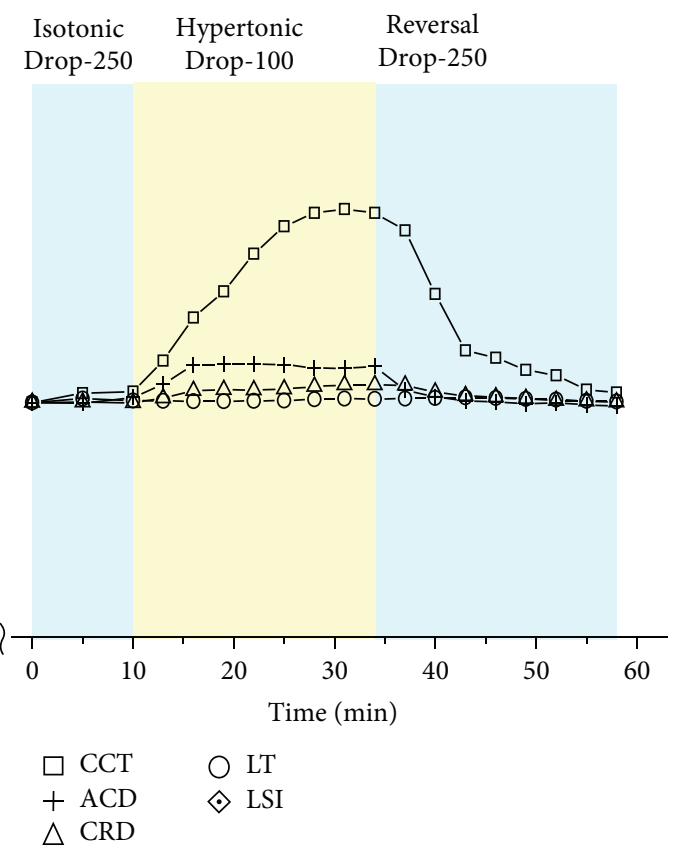

(d)

Figure 2: Normalized changes of cornea, lens, and retina in response to osmotic challenges. (a) Control group. (b) Hypertonic Drop-1000 challenge and reversal. (c) Hypertonic Drop-500 challenge and reversal. (d) Hypotonic Drop-100 challenge and reversal.

closing of the iridocorneal angle (Figure 3) in the hypertonic challenges might be correlated with the development of cataract $[4,7,8]$ and glaucoma [9-11], respectively. Thus, the hydration control is an important physiological process and might have a significant impact on almost all the ocular components, including the cornea, the iris, the lens, and the retina. OCT may offer a unique capability to quantitatively study the dynamic responses.

Rodent is one of the most frequently adopted model for improved understanding of disease pathogenesis and development of novel therapies $[39,40]$. This study demonstrate that the hydration condition would significantly influence the 


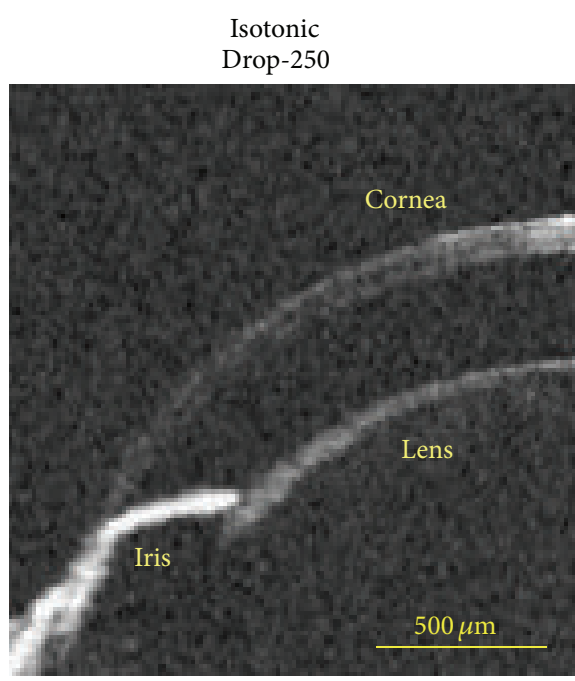

(a)

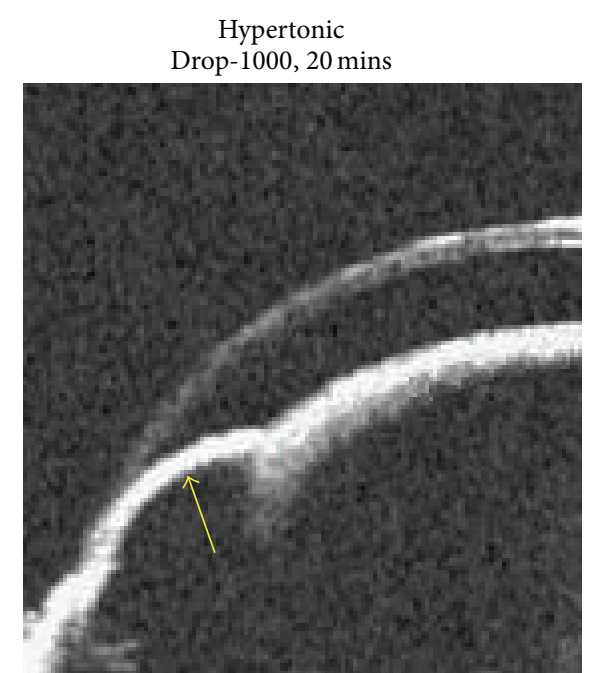

(b)

Hypotonic

Drop-100, 20 mins

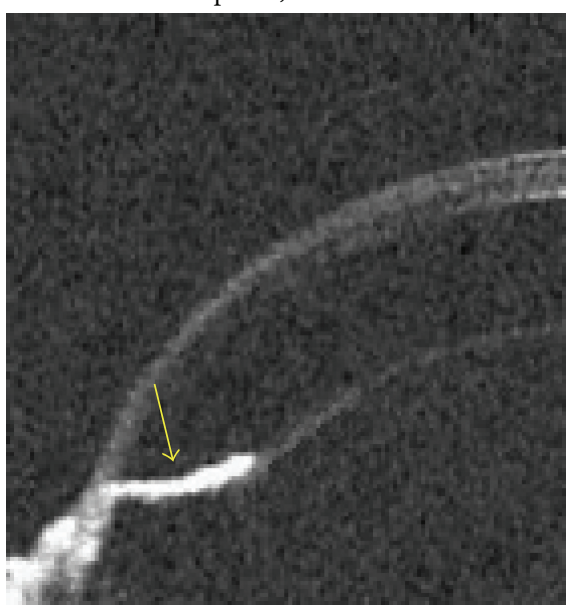

(c)

FIGURE 3: Iris response to osmotic challenges indicating the IOP change. (a) Isotonic phase. (b) Hypertonic phase at the time instant of 20 mins after the administration of Drop-1000 challenge. (c) Hypotonic phase at the time instant of 20 mins after the administration of Drop100 challenge. The bold arrows indicate the possible direction of the IOP-induced force that acted on the iris.

morphological architecture of the mouse eye and may lead to a great variance in the biometric measurements. Moreover, the lens opacification appearing during the hypertonic challenge may have a great influence on the OCT image quality. As shown in Figure 1(c), the OCT intensity of the retina is apparently weak in the hypertonic phase compared with the isotonic phases. In brief, attentions should be paid to the impact of hydration control for higher measuring accuracy and imaging quality of the eye.

\section{Conclusion}

The osmotic challenge could induce a significant impact on the morphology of almost all the ocular components, including the cornea, the lens, and the retina, and the light transparency of the lens. Thus, the ocular hydration is an important physiological process, which might be correlated with various ocular disorders, such as dry eye, cataract, and glaucoma. Moreover, our results indicate that additional attentions should be paid on hydration control during the biometric measurement and fundus imaging. OCT offers a unique capability to quantitatively study the dynamic full-eye responses to osmotic stress in vivo.

\section{Conflict of Interests}

The authors declare that there is no conflict of interests regarding the publication of this paper.

\section{Acknowledgments}

This work was supported by the National Natural Science Foundation of China (61475143, 11404285, 61335003, 
81200717, 61327007, and 61275196); National Hi-Tech Research and Development Program of China (2015AA020515); Zhejiang Provincial Natural Science Foundation of China (LY14F050007); Zhejiang Province Science and Technology Grant (2015C33108); Fundamental Research Funds for the Central Universities (2014QNA5017); Scientific Research Foundation for Returned Scholars, Ministry of Education of China.

\section{References}

[1] N. P. Walsh, M. B. Fortes, and M. Esmaeelpour, "Influence of modest changes in whole-body hydration on tear fluid osmolarity: important considerations for dry eye disease detection," Cornea, vol. 30, no. 12, pp. 1517-1518, 2011.

[2] N. P. Walsh, M. B. Fortes, P. Raymond-Barker et al., "Is wholebody hydration an important consideration in dry eye?" Investigative Ophthalmology and Visual Science, vol. 53, no. 10, pp. 6622-6627, 2012.

[3] D. L. Williams and B. K. Mann, "A crosslinked HA-based hydrogel ameliorates dry eye symptoms in dogs," International Journal of Biomaterials, vol. 2013, Article ID 460437, 8 pages, 2013.

[4] J. H. Kinoshita and L. O. Merola, "Hydration of the lens during the development of galactose cataract," Investigative Ophthalmology, vol. 3, pp. 577-584, 1964.

[5] B. W. Lambert and J. H. Kinoshita, "The effects of ionizing irradiation on lens cation permeability, transport, and hydration," Investigative Ophthalmology, vol. 6, no. 6, pp. 624-634, 1967.

[6] A. Mizuno, S. Toshima, and Y. Mori, "Confirmation of lens hydration by Raman spectroscopy," Experimental Eye Research, vol. 50, no. 6, pp. 647-649, 1990.

[7] M. Ø. Jensen, R. O. Dror, H. Xu et al., "Dynamic control of slow water transport by aquaporin 0 : implications for hydration and junction stability in the eye lens," Proceedings of the National Academy of Sciences of the United States of America, vol. 105, no. 38, pp. 14430-14435, 2008.

[8] K. Tompa, P. Bánki, M. Bokor, P. Kamasa, P. Rácz, and P. Tompa, "Hydration water/interfacial water in crystalline lens," Experimental Eye Research, vol. 91, no. 1, pp. 76-84, 2010.

[9] F. Bigar and R. Witmer, "Corneal endothelial changes in primary acute angle-closure glaucoma," Ophthalmology, vol. 89, no. 6, pp. 596-599, 1982.

[10] F. Manz, "Hydration and disease," Journal of the American College of Nutrition, vol. 26, no. 5, supplement, pp. 535S-541S, 2007.

[11] F. S. Ali and E. K. Akpek, "Glaucoma and dry eye," Ophthalmology, vol. 116, no. 6, article 1232, 2009.

[12] G. Simon, R. H. Small, Q. Ren, and J.-M. Parel, "Effect of corneal hydration on Goldmann applanation tonometry and corneal topography," Refractive and Corneal Surgery, vol. 9, no. 2, pp. 110-117, 1993.

[13] M. J. Doughty and M. L. Zaman, "Human corneal thickness and its impact on intraocular pressure measures: a review and metaanalysis approach," Survey of Ophthalmology, vol. 44, no. 5, pp. 367-408, 2000.

[14] S. C. Pflugfelder, A. Solomon, and M. E. Stern, "The diagnosis and management of dry eye: a twenty-five-year review," Cornea, vol. 19, no. 5, pp. 644-649, 2000.

[15] I. G. Pallikaris, H. S. Ginis, G. A. Kounis, D. Anglos, T. G. Papazoglou, and L. P. Naoumidis, "Corneal hydration monitored by laser-induced breakdown spectroscopy," Journal of Refractive Surgery, vol. 14, no. 6, pp. 655-660, 1998.

[16] N. J. C. Bauer, J. P. Wicksted, F. H. M. Jongsma, W. F. March, F. Hendrikse, and M. Motamedi, "Noninvasive assessment of the hydration gradient across the cornea using confocal raman spectroscopy," Investigative Ophthalmology and Visual Science, vol. 39, no. 5, pp. 831-835, 1998.

[17] J. Fischbarg and D. M. Maurice, "An update on corneal hydration control," Experimental Eye Research, vol. 78, no. 3, pp. 537541, 2004.

[18] J. W. Ruberti and S. D. Klyce, "NaCl osmotic perturbation can modulate hydration control in rabbit cornea," Experimental Eye Research, vol. 76, no. 3, pp. 349-359, 2003.

[19] J. M. Marcantonio, G. Duncan, and H. Rink, "Calcium-induced opacification and loss of protein in the organ-cultured bovine lens," Experimental Eye Research, vol. 42, no. 6, pp. 617-630, 1986.

[20] K. R. Hightower and M. Dering, "Development and reversal of calcium-induced opacities in vitro," Investigative Ophthalmology and Visual Science, vol. 25, no. 9, pp. 1108-1111, 1984.

[21] P. Li, M. Johnstone, and R. K. Wang, "Full anterior segment biometry with extended imaging range spectral domain optical coherence tomography at $1340 \mathrm{~nm}$," Journal of Biomedical Optics, vol. 19, no. 4, Article ID 046013, 2014.

[22] L. Wang, B. Hofer, Y.-P. Chen, J. A. Guggenheim, W. Drexler, and B. Považay, "Highly reproducible swept-source, dispersionencoded full-range biometry and imaging of the mouse eye," Journal of Biomedical Optics, vol. 15, no. 4, Article ID 046004, 2010.

[23] M. Jiang, P.-C. Wu, M. E. Fini et al., "Single-shot dimension measurements of the mouse eye using SD-OCT," Ophthalmic Surgery Lasers and Imaging, vol. 43, no. 3, pp. 252-256, 2012.

[24] H. N. Park, Y. Qazi, C. Tan et al., "Assessment of axial length measurements in mouse eyes," Optometry \& Vision Science, vol. 89, no. 3, pp. 296-303, 2012.

[25] I. Grulkowski, J. J. Liu, B. Potsaid et al., "Retinal, anterior segment and full eye imaging using ultrahigh speed swept source OCT with vertical-cavity surface emitting lasers," Biomedical Optics Express, vol. 3, no. 11, pp. 2733-2751, 2012.

[26] M. G. Ghosn, V. V. Tuchin, and K. V. Larin, "Depth-resolved monitoring of glucose diffusion in tissues by using optical coherence tomography," Optics Letters, vol. 31, no. 15, pp. 23142316, 2006.

[27] M. G. Ghosn, V. V. Tuchin, and K. V. Larin, "Nondestructive quantification of analyte diffusion in cornea and sclera using optical coherence tomography," Investigative Ophthalmology and Visual Science, vol. 48, no. 6, pp. 2726-2733, 2007.

[28] K. Hosseini, A. I. Kholodnykh, I. Y. Petrova, R. O. Esenaliev, F. Hendrikse, and M. Motamedi, "Monitoring of rabbit cornea response to dehydration stress by optical coherence tomography," Investigative Ophthalmology and Visual Science, vol. 45, no. 8, pp. 2555-2562, 2004.

[29] P. Li, Z. Ding, Y. Ni et al., "Visualization of the ocular pulse in the anterior chamber of the mouse eye in vivo using phasesensitive optical coherence tomography," Journal of Biomedical Optics, vol. 19, no. 9, Article ID 090502, 2014.

[30] P. Li, L. An, G. Lan, M. Johnstone, D. Malchow, and R. K. Wang, "Extended imaging depth to $12 \mathrm{~mm}$ for $1050-\mathrm{nm}$ spectral domain optical coherence tomography for imaging the whole anterior segment of the human eye at $120-\mathrm{kHz}$ A-scan rate," Journal of Biomedical Optics, vol. 18, no. 1, Article ID 016012, 2013. 
[31] R. A. Leitgeb, R. Michaely, T. Lasser, and S. Chandra Sekhar, "Complex ambiguity-free Fourier domain optical coherence tomography through transverse scanning," Optics Letters, vol. 32, no. 23, pp. 3453-3455, 2007.

[32] B. Baumann, M. Pircher, E. Götzinger, and C. K. Hitzenberger, "Full range complex spectral domain optical coherence tomography without additional phase shifters," Optics Express, vol. 15, no. 20, pp. 13375-13387, 2007.

[33] L. An and R. K. Wang, "Use of a scanner to modulate spatial interferograms for in vivo full-range Fourier-domain optical coherence tomography," Optics Letters, vol. 32, no. 23, pp. 34233425, 2007.

[34] M. R. Hee, J. A. Izatt, E. A. Swanson et al., "Optical coherence tomography of the human retina," Archives of Ophthalmology, vol. 113, no. 3, pp. 325-332, 1995.

[35] C. Du, M. Shen, M. Li, D. Zhu, M. R. Wang, and J. Wang, "Anterior segment biometry during accommodation imaged with ultralong scan depth optical coherence tomography," Ophthalmology, vol. 119, no. 12, pp. 2479-2485, 2012.

[36] G. B. Benedek, "Theory of transparency of the eye," Applied Optics, vol. 10, no. 3, pp. 459-473, 1971.

[37] D. M. Maurice, "The structure and transparency of the cornea," The Journal of Physiology, vol. 136, no. 2, pp. 263-286, 1957.

[38] C. Y. Cheung, S. Liu, R. N. Weinreb et al., "Dynamic analysis of iris configuration with anterior segment optical coherence tomography," Investigative Opthalmology \& Visual Science, vol. 51, no. 8, pp. 4040-4046, 2010.

[39] C. P. Austin, J. F. Battey, A. Bradley et al., "The knockout mouse project," Nature Genetics, vol. 36, no. 9, pp. 921-924, 2004.

[40] M. T. Pardue, R. A. Stone, and P. M. Iuvone, "Investigating mechanisms of myopia in mice," Experimental Eye Research, vol. 114, pp. 96-105, 2013. 


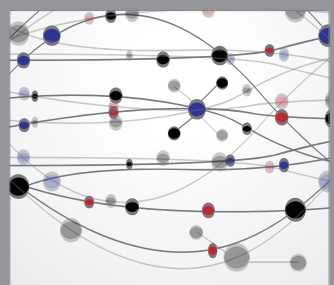

The Scientific World Journal
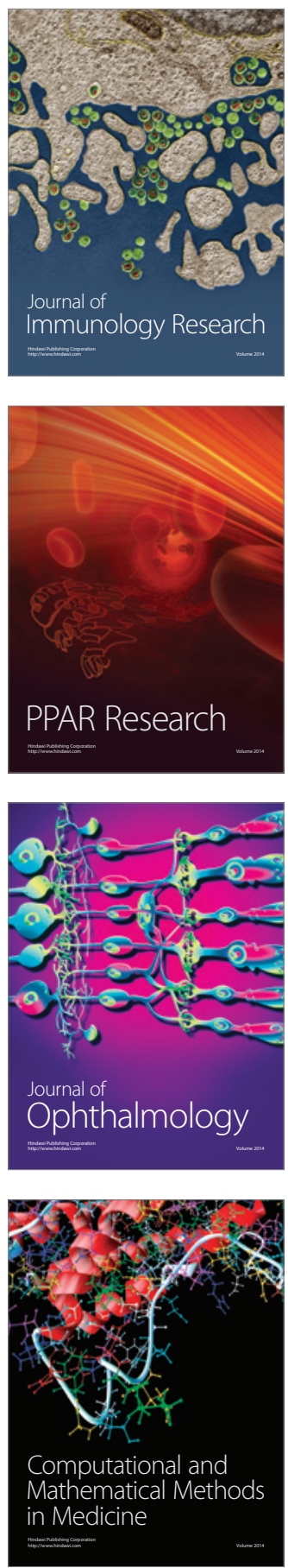

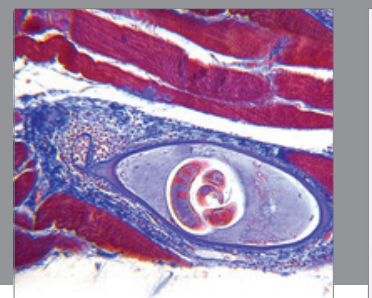

Gastroenterology

Research and Practice
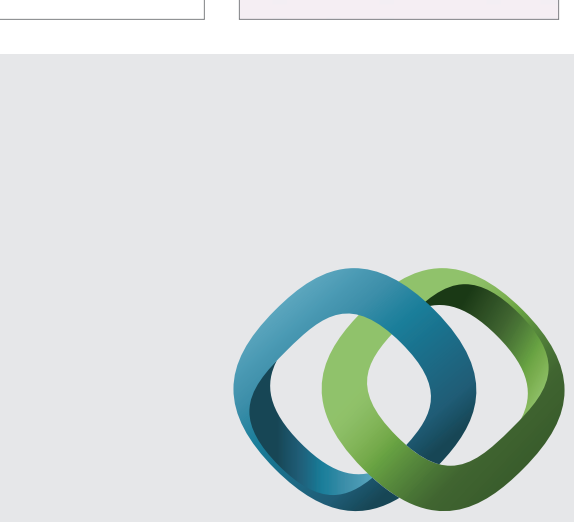

\section{Hindawi}

Submit your manuscripts at

http://www.hindawi.com
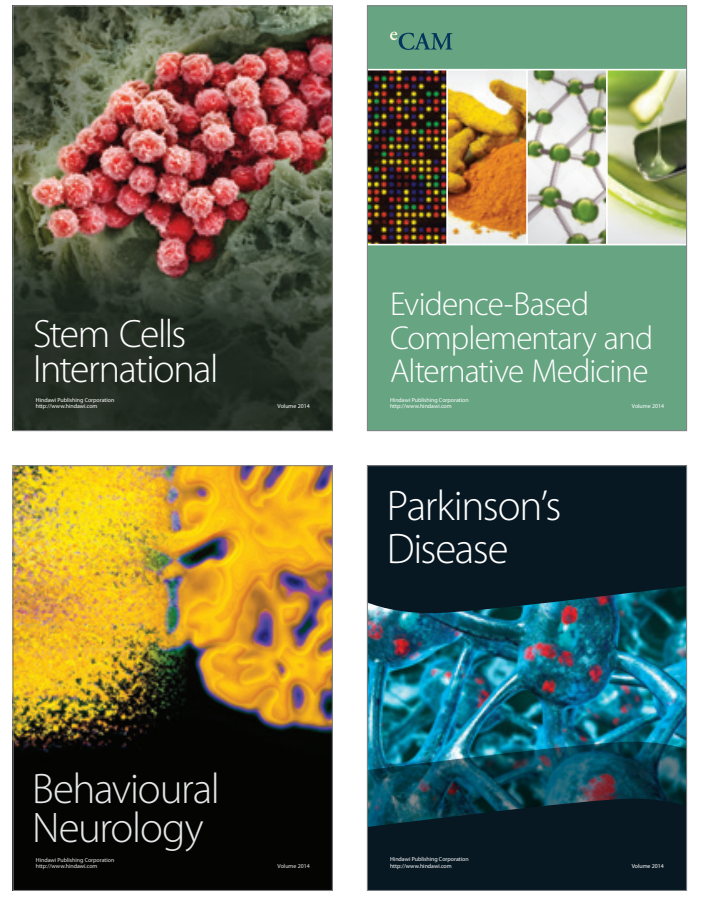
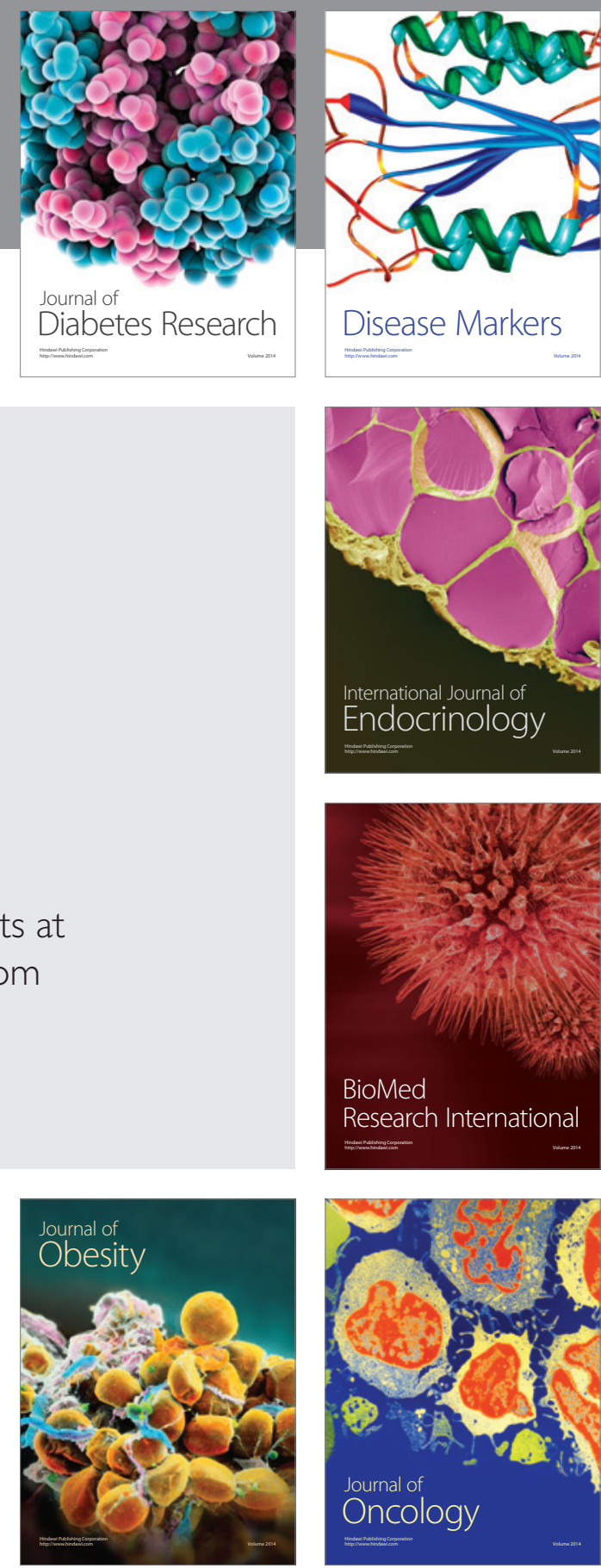

Disease Markers
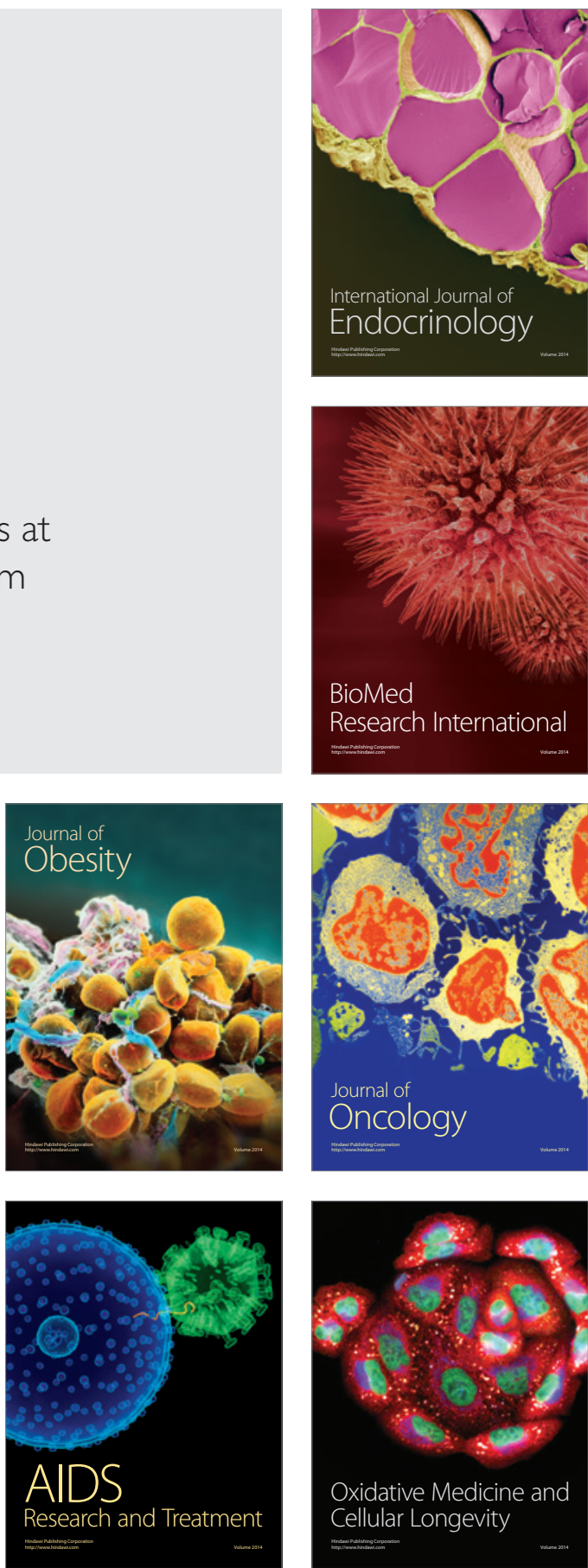